\title{
LA EFICACIA SIMBÓLICA DEL DESARROLLO: ESTRATEGIA DE CONSTRUCCIÓN DEL SUJETO HISTÓRICO
}

Matilde Eljach Universidad Simón Bolívar Colombia

Ezequiel Quiroz Universidad Simón Bolívar

Colombia 
Panorama Económico, Vol. 26 - No. 4 (Octubre - Diciembre de 2018), pp. 497-514

\author{
Matilde Eljach \\ Ezequiel Quiroz
}

\title{
La eficacia simbólica del desarrollo: estrategia de construcción del sujeto histórico
}

\begin{abstract}
Resumen
Este documento presenta una reflexión acerca de la pertinencia del concepto contemporáneo de desarrollo y su alcance en la práctica. La eficacia del concepto de desarrollo acuñado en los tiempos modernos es simbólica y se ve erosionada por las prácticas económicas que cuestionan su eficacia. Los sistemas económicos, políticos y culturales han propuesto sistemas simbólicos con cierto grado de exclusión. En diferentes etapas, surgen nuevos paradigmas sobre el desarrollo sin considerar a los individuos que más necesitan en el sistema capitalista. El concepto de desarrollo aún no considera la inclusión social, las prácticas "Buen Vivir", la participación colectiva, la conservación de recursos y la protección ambiental. En este sentido, los nuevos indicadores del desarrollo deben considerar los impactos ambientales y sociales.
\end{abstract}

Palabras clave: Desarrollo, Bienestar, Modernidad.

Clasificación JEL: B50, O15

The symbolic development effectiveness: strategy of construction of the historical subject

\begin{abstract}
The concept of development has been coined in modern times, and its effectiveness is symbolic and is eroded by economic practices that question its efficacy. The economic, political and cultural systems have proposed symbolic systems with some degree of exclusion. At different stages, new paradigms about development emerge without considering those individuals that need most in the capitalist system. The concept of development does not consider yet social inclusion, "Buen Vivir" practices, collective participation, resources conservation, and environmental protection. In this sense, development as a framework, should consider environmental and social impacts.
\end{abstract}

Keywords: Development, Wellbeing, Modernity.

JEL Classification: B50, O15

\section{L'efficacité symbolique du développement: stratégie de construction du sujet historique}

\section{Résumé}

Le concept de développement a été inventé à l'époque moderne et son efficacité est symbolique et érodée par des pratiques économiques qui remettent en question son efficacité. Les systèmes économique, politique et culturel ont proposé des systèmes symboliques avec un certain degré d'exclusion. À différents stades, de nouveaux paradigmes sur le développement apparaissent sans tenir compte des individus qui ont le plus besoin du système capitaliste. Le concept de développement ne prend pas encore en compte l'inclusion sociale, les pratiques "Buen Vivir", la participation collective, la conservation des ressources et la protection de l'environnement. En ce sens, le développement en tant que cadre devrait prendre en compte les impacts environnementaux et sociaux.

Mots-clés: Développement, Bien-être, Modernité.

Nomenclature JEL: B50, O15 


\section{La eficacia simbólica del desarrollo: estrategia de construcción del sujeto histórico}

INFORMACIÓN DEL ARTÍCULO

Recepción de artículo: 14/07/2017

Concepto de evaluación: 23/08/2017

Aceptación de artículo: 19/11/2017
Matilde Eljach*

Universidad Simón Bolívar

Colombia

Ezequiel Quiroz

Universidad Simón Bolívar

Colombia

\section{INTRODUCCIÓN}

El presente artículo busca relacionar el papel que juega el discurso -en este caso el discurso sobre el concepto de desarrollo-, con los resultados evidentes del mismo. Evidenciar cómo el poder económico y político construye con dispositivos retóricos al "otro", al diferente; lo nomina, lo resignifica, lo reconstruye, para naturalizar sus requerimientos e imponerlos como exigencias de vida de los nominados -dominados- a través de políticas de desarrollo expresadas en el universalismo discursivo del logos binario de la modernidad, la cual crea un espacio-tiempo único, universal, el de la filosofía de la historia hegeliana.

Contrastándolo con experiencias de vida que privilegian otras formas de ser y estar en el mundo; diversas prácticas de entendimiento e integración con la naturaleza. Prácticas que riñen con los modelos de desarrollo hegemónicos y que se asientan en valores construidos en colectividad, como la experiencia de Bután y de "El Buen Vivir" de los pueblos originarios indoamericanos, por ejemplo. En ese sentido el documento cuestiona el concepto de desarrollo heredado de la modernidad y su eficacia simbólica e indaga por otras construcciones como bienestar y felicidad.

¿Qué es felicidad, bienestar, desarrollo y como medirlos? ¿Son sinónimos esos términos? Los grandes filósofos de la historia antigua se preocuparon por definir la felicidad. Tales de Mileto (625-547 a. C.) decía: "quien tiene un cuerpo sano, fortuna y un alma bien educada".

\footnotetext{
* Autor para correspondencia

Correos electrónicos: matilde.eljach@unisimonbolivar.edu.co*, equiroz@unisimonbolivar.edu.co
} 
Demócrito Abdera (460- 370 a.C.) desde esa época trataba definir y medir la felicidad como: "la medida del placer y la proporción de la vida. O sea como el mantenerse alejado de todo defecto y de todo exceso"; Aristóteles de Estagira (384-322 a. C.): "la felicidad debe ser el propósito de la vida". Esto ha sido, no solo preocupación de los grandes pensadores y filósofos de la Edad Antigua y de la Edad Media, sino de los científicos sociales de la edad Moderna y Contemporánea, donde el término felicidad del hombre ha mutado hacia el concepto de "bienestar" o "desarrollo social", "Bien Vivir" o "Vivir Bien". Thomas Jefferson (1743-1826) tercer presidente de Estados Unidos de América refiriéndose al mismo tema: "no son las riquezas ni el esplendor, sino la tranquilidad y el trabajo, los que proporcionan la felicidad". Eduardo Gudynas, prestigioso ambientalista uruguayo investigador del Centro Latino Americano de Ecología Social CLAES), expone el pensamiento de los pueblos originarios indoamericanos cuando dice: "Bien Vivir o Vivir Bien, engloba un conjunto de ideas que se están forjando como reacción y alternativas a los conceptos convencionales sobre el desarrollo". Es un concepto en construcción que se está germinando en distintas posturas en distintos países con diversos actores sociales, principalmente en Ecuador y Bolivia basado en la cosmovisión de los pueblos indígenas en la construcción del concepto de lo que podría denominarse mejoramiento social.(Gudynas E, 2011, pag. 232).

Durante todo el siglo XIX, se consideró la importancia de la economía del bienestar y que los economistas no solo deberían explicar el mundo económico tal como es y como ha sido, y formular pronósticos sobre el curso futuro de los hechos económicos, sino también establecer principios de política económica que conlleven a promover el bienestar social.

Entendido el desarrollo como el grado de bienestar de los individuos de una sociedad, la discusión, se centra en cómo medir ese grado de bienestar, sin la exclusión del otro, con otra cultura, en otro tipo de economía.

Existen indicadores económicos y sociales elaborados por organismos internacionales, que se han utilizado para medir el crecimiento económico y el grado de desarrollo social de los países. Entre estos indicadores, están los carácter estrictamente económico, como el Producto Interno Bruto (PIB) per cápita y el Ingreso Nacional per cápita (INP) que hacen, referencia al valor de la producción total de bienes y de servicios y a los ingresos nacionales generados en una economía, divididos por la población del país. Estos indicadores han resultado los más vulnerables a las críticas que se formulan por parte de estudiosos del tema por sus limitaciones para medir aspectos sociales de bienestar o de desarrollo de un país. Amartya Sen, premio nobel de Economía (1988) afirma en su libro "Desarrollo y Libertad", que: "el desarrollo puede concebirse como un proceso de expansión de las libertades reales de que disfrutan los individuos". El desarrollo para él no es un fin en sí mismo, sino un medio para llegar a una meta: la felicidad de los individuos. Entiende este desarrollo como un proceso de expansión de las libertades reales de los individuos, que contrasta con las versiones tradicionales de desarrollo como el crecimiento del producto nacional bruto y de las rentas personales, con la industrialización y con la modernización social, conceptos éstos que pueden contribuir como medio a la expansión de las libertades, pero que por sí solos, no pueden ser considerados como desarrollo. El desarrollo que Sen plantea exige libertad económica y política de los individuos y la eliminación de las principales fuentes de privaciones de libertad: la pobreza y la tiranía, la escasez de oportunidades económicas y 
las privaciones sociales sistemáticas como el abandono de los servicios públicos en educación y salud y la intolerancia y de las restricciones de las libertades políticas y civiles para participar en vida social, política y económica de la sociedad. (Sen A, 2000, pag 3).

El Informe de Desarrollo Humano (1990) publicado por el PNUD presenta una visión más integral sobre desarrollo humano a través del Índice de Desarrollo Humano(IDH), elaborado con base al enfoque de las capacidades y titularidades que Amartya Sen considera deben tener las personas, no solo de las posibilidades de elección que permitan una mayor ingreso, sino de oportunidades razonables para desarrollar potencialidades, para un mejor nivel de vida. Este índice se enfoca en tres aspectos fundamentales: longevidad, conocimiento y nivel de vida decente, perfilados en variables sobre capacidad del talento humano, esperanza de vida, salud, educación, libertad, pobreza, distribución del ingreso e inequidad, entre muchas otras variables; sin embargo, a pesar de que este indicador contiene un enfoque más humano del desarrollo y sus indicadores derivados sobre Índice de Desarrollo de Genero (IDG), Índice de Desarrollo de la Mujer (IPM), Índice de Pobreza Humana (IPH), entre otros subíndices importantes, se le critica porque mezcla "indicadores de resultados" como esperanza de vida, nutrición, alfabetismo, con "variables de insumos", como acceso a agua potable y servicios de salud, en consecuencia, existen críticas sobre si mide la pobreza no monetaria o el acceso servicios públicos para reducir la pobreza. En mi concepto muy a pesar de esta interpretación y otras críticas que se le han hecho sobre la arbitrariedad en la selección y ponderación de las variables de privación, en la calidad de los datos y de fuentes utilizados en algunos países, nada más valioso que haber suscitado el interés general hacia el desarrollo humano y la vasta lista de indicadores que se derivan de él; sin desconocer además que el PIB per cápita como indicador del desarrollo aun se sigue utilizando, sin olvidar que ninguno de ellos dan cuenta perfectamente del desarrollo humano como lo sostiene Xavier Mancero en un documento de la CEPAL titulado: La medición del desarrollo humano: elementos de debate" (CEPAL , 2001, pag. 36)

Existen otros indicadores que se abren paso dentro de la gama ya existente y que se elaboran con metodologías novedosas y menos complejas combinando, variables objetivas ya conocidas y variables subjetivas como la felicidad, la libertad, la preocupación, el estrés, entre otras. Estos indicadores son conocidos como Medidas de Bienestar Subjetivo, finalmente están los indicadores de sostenibilidad ambiental que incluyen variables de impacto sobre el medio ambiente, y se encuentran en una fase muy preliminar

En el Informe Brundtland (1987) preparado por Naciones Unidas se menciona por primer vez el términos de "desarrollo sostenible o sustentable", nuevo paradigma de las ciencias sociales, con un concepto diferente al desarrollo industrialista tradicional. Dice el informe: "Está en manos de la humanidad hacer que el desarrollo sea sostenible, duradero, o sea, asegurar que satisfaga las necesidades del presente sin comprometer la capacidad de las generaciones futuras para satisfacer las propias" (Naciones Unidas, 1987, pp 89 ). Este concepto implica límites al uso indiscriminado de los recursos naturales y a su vez control de aquellas actividades humanas que afectan al medio ambiente, por la dificultad de la biosfera de absorber y recuperarse de la creciente emisión y vertimiento a la atmósfera de los gases de efecto invernadero desatado por el incremento de las actividades humanas y el desarrollo tecnológico y sus consecuencias en cambio climático. 
El nuevo concepto acuñado hace veinte años, en el Informe Brundtland, en honor a la Gro Harlem Brundtland, quien encabezaba la Misión de las Naciones Unidas encargada de elaborar dicho informe sobre Medio Ambiente y Desarrollo, titulado "Nuestro Futuro Común", ante el rumbo que tomaba el mundo frente a la sociedad global que agrede cada vez más los recursos naturales del planeta y hace más vulnerable a la población más pobre, ha hecho que surjan nuevas metodologías e indicadores para medir el desarrollo, ahora no solo en la parte cuantitativa de la economía sino de sus efectos sobre la sociedad y sobre la naturaleza. Este nuevo paradigma del desarrollo hace que surjan nuevos diseños metodológicos e indicadores simples y compuestos que tratan de medir el desarrollo. Como el Índice de desarrollo Sostenible (IBES) o por sus siglas en inglés ISEW) que contiene variables económicas, sociales y ambientales y mide la dinámica de crecimiento bajo la óptica de la sostenibilidad ambiental; la huella ecológica definida como el uso de espacio ambiental (nacional, regional o per cápita) necesario para producir los niveles de vida que existen en determinados asentamientos humanos; el Índice de sostenibilidad Ambiental (ISA), que contempla una cantidad apreciables de indicares ambientales.

En este orden de ideas es importante presentar el caso de Bután reseñado por Pablo Guimón en una breve crónica sobre "El reino que quiso medir la felicidad", relatando que en Bután, aislado reino del Himalaya, con una población menor a un (1) millón de habitantes se construyó la democracia más joven del mundo, sin requerir los procesos sangrientos que en muchos países han sido indispensables, violentando la concepción dominante del pensamiento económico global. El rey Jigme Singye Wangchuck, amado como el rey dios por su pueblo, "acuñó, hace 35 años, un término que hoy, en este escenario del poscomunismo y del post capitalismo salvaje, constituye el centro de uno de los debates más interesantes que se están produciendo en el pensamiento económico mundial. Un debate al que se han apuntado premios Nobel como Joseph E. Stiglitz o Amartya Sen y líderes occidentales como Nicolás Sarkozy o Gordon Brown (....): La Felicidad Interior Bruta (FIB) es mucho más importante que el Producto Interior Bruto". (Guimón, 2009, elpais.com/diario/2009/11/29/ eps/1259479614_850215.html).

Esta frase en apariencia simple, expresa la concepción profundamente diferente de la política y del modelo de desarrollo de Bután, una problematización de la economía y de la política desde una concepción moral diferente; economía alterna al capitalismo que es un espacio-tiempo otro al Estado nación y a la modernidad, porque lo económico no puede reducirse al capital (Bataille, 1993); es la irrupción de otra economía en la praxis política: "(...) La idea es que el modo de medir el progreso no debe basarse estrictamente en el flujo de dinero. El verdadero desarrollo de una sociedad, defienden, tiene lugar cuando los avances en lo material y en lo espiritual se complementan y se refuerzan uno a otro. Cada paso de una sociedad debe valorarse en función no sólo de su rendimiento económico, sino de si conduce o no a la felicidad." (Guimón, 2009, elpais.com/diario/2009/11/29/ eps/1259479614_850215.html).

Las condiciones básicas para lograr la Felicidad Interna Bruta (FIB), que constituye el eje del modelo de desarrollo en Bután, han sido la tradición budista y el tardío acceso a la modernidad, lo que explicado en términos de E.P. Thompson, constituye una moral otra que desarma la unicidad de lo económico, que hace naufragar el valor de uso y el valor de 
cambio (Thompson, 1995). Y en tanto no todo es mercancía, Bután ejemplifica que no hay mercado sin cultura, sin lucha simbólica:

(...) en 2007 Bután fue la segunda economía que más rápido creció en el mundo. La educación, gratuita y en inglés, llega hoy a casi todos los rincones del país. En un estudio realizado en 2005, el 45\% de los butaneses declaró sentirse "muy feliz", el 52\% reportó sentirse "feliz" y sólo el 3\% dijo no ser feliz. En el Mapamundi de la Felicidad, una investigación dirigida por el profesor Adrian White en la Universidad de Leicester (Reino Unido) en 2006, Bután resultó ser el octavo más feliz de los 178 países estudiados (por detrás de Dinamarca, Suiza, Austria, Islandia, Bahamas, Finlandia y Suecia). Y era el único entre los 10 primeros con un PIB per cápita muy bajo (5.312 dólares en 2008, seis veces menor que el español).

Este relato podría ser meramente anecdótico por fuera de la recurrente preocupación respecto a la situación económica de los países, en particular a la concepción sobre el concepto occidental de desarrollo que impera, y a los desacertados resultados del modelo de desarrollo que aplican si se tienen en cuenta los índices de desempleo, analfabetismo, insalubridad y de pobreza, entre otros; el discurso del desarrollo como manifestación de la violencia instrumentalizada por el orden (Anderson, 1998).

Boaventura de Sousa Santos (2011) afirma que en el mundo occidental hay dos tipos de pensamiento que han primado en América Latina y el Caribe y que tratan de dar explicación al status quo político y económico: el pensamiento triunfalista burgués y el pensamiento pesimista crítico eurocéntrico. Ambos pensamientos de discurso excluyentes, uno más que el otro, pues niegan al desarrollo histórico, económico y social del "otro" y los derechos de la naturaleza. Contrario a estos pensamientos hay otras alternativas prácticas de pensamiento incluyentes sobre desarrollo que no se visibilizan, ni son creíbles por nuestra manera de pensar. La praxis del pensamiento eurocéntrico actualmente coexiste con la época de una modernidad que puede ser superada desde otra epistemología de corte histórica y cultural, que reconozca la relación ecosistema del hombre con la naturaleza. Se vale de Sousa, al igual de Eduardo Gudynas, de los procesos políticos de Bolivia y Ecuador para la redefinición de conceptos : Buen Vivir, Estado, plurinacionalidad para demostrar que existen en estos países intensos movimientos políticos de origen indigenistas que han posibilitado nuevas y sendas Constituciones Políticas Ecuador (2008) y Bolivia (2009), altamente innovadoras que contienen concepciones alternativas sobre el Estado (plurinacionalidad, democracia participativa), del desarrollo (SunaK Kawsav) y de los derechos humanos y de la naturaleza.

Arturo Escobar presenta un análisis exhaustivo sobre los modelos de desarrollo que se han "vendido" a América Latina y al Tercer Mundo en general; así como de Sousa, Aníbal Quijano, Eduardo Gudynas, entre otros, han tratado de refundar el concepto de Estado, de desarrollo y del Buen Vivir, a partir de experiencias que aunque han surgido en el sur de América Latina son válidas en el norte del continente y en otras partes del mundo. Esta crítica apunta a cómo las estrategias desarrollistas no sólo niegan al otro, al desconocer sus historias y procesos económicos, sociales y culturales, sino que los despoja de la 
autonomía y determinación requeridas para orientar sus destinos hacia los rumbos que las comunidades establezcan.

\section{II}

En "La invención del Tercer Mundo. Construcción y deconstrucción del desarrollo" (2004), el investigador Arturo Escobar, expone las estrategias que desplegó la segunda posguerra, para "descubrir" Asia, África y América Latina, con el propósito de generar -amparados en un afán mesiánico/altruista "de que había que hacer algo antes de que los niveles de inestabilidad en el mundo entero se volvieran intolerables" (Escobar, 2004:52) - una restructuración de la cultura y la economía globales, para protegerse de la nueva amenaza global. El interés bélico se desplazó al interés social por el que fue entonces llamado Tercer Mundo, y surgió un nuevo enemigo: la pobreza del Tercer Mundo, y contra éste se acometió un nuevo tipo de guerra, definiendo un nuevo orden social y político.

Señala Escobar que desde el siglo XIX el interés por lo social cobró importancia, propiciando la aparición del Estado benefactor y consolidando el concepto de trabajo social. Se naturalizó una nueva concepción de seres humanos y sociales, enmarcados en la categoría de "pobres". Y, esta antesala facilitó que después de 1945, segunda posguerra, tal categoría se generalizara, globalizando a los habitantes de las dos terceras partes del mundo en el concepto de "pobres", y territorializando el concepto con la fábula del Tercer Mundo. ¿Quiénes fueron los "pobres" desde entonces?, al respecto señala Escobar:

"La fábula de los tres mundos fue, y sigue siendo a pesar de la defunción del segundo, una manera de crear un orden político que "funciona mediante la negociación de fronteras logradas a través del ordenamiento de las diferencias" (Haraway, 1989: 10). Fue (y es) una narrativa donde cultura, raza, género, nación y clase están inextricablemente ligadas. El orden político y económico codificado por la fábula de los tres mundos y el desarrollo descansa sobre el tráfico de significados que describen nuevos campos del ser y del entender, los mismos campos que son cada vez más cuestionados y desestabilizados por las gentes del Tercer Mundo hoy en día" (Escobar, 2004: 56)

Aunque los conceptos de "pobres" y "pobreza", "ricos" y "riqueza" son muy antiguos y se remontan al Antiguo Testamento desde cuando el pueblo de Israel pasó de una vida seminómada a sedentaria, y la lucha contra la pobreza está presente en todo el evangelio, los "pobres" y los "ricos", han estado presente en los distintos modus de producción pre, capitalista y post capitalista: esclavitud, feudalismo, capitalismo, globalización y modernidad. El concepto de "pobres", "esclavos", "siervos", "obreros", "indígenas", "desechables", "mendigos", entre otros términos, se han utilizado para caracterizar a los desposeídos de bienes materiales y de la riqueza, sin alcanzar los niveles de vida deseados. En la sociedad contemporánea la lucha contra la pobreza es considerada como política esencial para preservar el statu quo de la sociedad desarrollista capitalista en los países tercermundistas de América Latina y del resto del planeta. 
A partir de entonces, el Primer Mundo comienza a desplegar sus estrategias, inventando necesidades y nominando las nuevas realidades concebidas y creadas por él mismo. A saber: pobres, Primer Mundo, Segundo Mundo, Tercer Mundo, país subdesarrollado, escenario "desesperado e infeliz", anormal, iletrados, subdesarrollados, malnutridos, pequeños agricultores, campesinos sin tierra, países menos desarrollados, analfabetismo, hambre, indios, negros, marginales, mujeres lactantes, etc.

El "otro", el pobre, desnutrido, etc., es una construcción política, históricamente inscrita en relaciones de jerarquía y de poder, en los términos de Foucault (1999); que como construcción social, responde al reconocimiento de las propias negaciones y temores, o a la estigmatización de la propia "naturaleza", de lo que no se quisiera llegar a ser; en el caso que ocupa, el temor inocultable del Primer Mundo a la amenaza de los desposeídos de la tierra que pudieran atentar contra sus mejores condiciones de vida, fustigados posiblemente por el comunismo. ¿Se comienza a proyectar la llamada guerra preventiva?

O bien, de aquellos "atributos", o valoraciones que se desean, o que se necesitan, o que idealmente se otorga; es decir, aquellos recursos naturales, culturales y sociales que en el mediano y largo plazo se podrían necesitar para sostener determinados niveles de crecimiento y desarrollo.

El discurso, en su sentido amplio, crea significados a partir de situaciones, que no son solo relativos, sino que están inscritos en la puesta en escena de la interlocución impregnada de identidades históricas y sociopolíticas. Interpretar los discursos y la interlocución en que se hacen manifiestos, implica prestar atención a la mutua asimilación de las historias identidades de los actores del discurso, lo cual propiciará que la interpretación determine los significados desde las propias hermenéuticas de los hablantes y oyentes. (Eljach, 2006).

Las diversas formas de exclusión: hambriento, descalzo, analfabeta, desnutrido, pobre, etc., lo que Amartya Sen llamaría las fuentes de las privaciones de las libertades del ser humano, constituyeron los límites y las fronteras de participación en la nueva distribución geopolítica del territorio, sin indagar por los procesos de construcción y de respuesta que el supuesto subdesarrollado creó como sus propios sistemas culturales y por las respuestas de orden económico, social y cultural que se generaron a lo largo de la historia para enfrentar sus propios requerimientos. Escobar, citando a Sachs (1990), a Rahnema (1991), Adas (1989), plantea lo siguiente:

“(...) como señalan los mismos autores dentro de las sociedades asiáticas, africanas, latinoamericanas o norteamericanas nativa, igual que a través de la mayor parte de la historia europea, las sociedades tradicionales habían desarrollado maneras de definir y tratar la pobreza que daban cabida a conceptos de comunidad, frugalidad y suficiencia. Como quiera que fueran tales formas tradicionales, y sin idealizarlas, es cierto que la pobreza masiva en el sentido moderno solamente apareció cuando la difusión de la economía de mercado rompió los lazos comunitarios y privó a millones de personas del acceso a la tierra, al agua y a otros recursos. Con la consolidación del capitalismo, la pauperización sistémica resultó inevitable" (Escobar, 2004: 52-53). 
De allí que primero se concibe la necesidad, se la construye y se la normaliza, generando lo que Escobar define como la burocratización de lo social. Lo social pensado y definido desde el escritorio, de espaldas a la realidad. Es una relación colonizadora, provocadora; en ese sentido no es inocente del poder. El poder no solo pervierte los intereses de la colectividad; también crea, produce. La alteridad está atravesada por concepciones y relaciones de poder.

Es una regularidad que solo se define en sociedad como expresión cultural; y su posibilidad expansiva está dada por las múltiples interpretaciones que pueda generar (Marc Augé, 1996). Es así como esa realidad para el análisis que nos ocupa, se expresa en las políticas y planes de desarrollo.

III

Implantada la estrategia discursiva de creación de la pobreza en el Tercer Mundo, es inevitable la estrategia salvadora de esa misma situación. El poder económico ya ha definido la necesidad, ahora debe definir la solución. Las Naciones Unidas plantea en 1948 que "La verdadera prosperidad mundial es indivisible" (Escobar, 2004: 52), lo cual ingenuamente podría entenderse como que todo el mundo participará de los mismos niveles de prosperidad, generando la verdadera avalancha de conocimiento científico, y de la conciencia política hacia el hambre mundial.

\begin{abstract}
"Semejante hambre de lenguaje (científico) ha traído como resultado una sucesión de diversas estrategias a lo largo de la era del desarrollo: desde los alimentos enriquecidos y los suplementos alimenticios pasando por la educación en nutrición y la ayuda alimentaria de los cincuenta y sesenta, hasta la reforma agraria, la revolución verde, el desarrollo rural integrado y la planificación alimentaria y nutricional exhaustiva, de finales de los sesenta, los lenguajes del hambre se han vuelto cada vez más detallados y de mayor alcance" (Escobar, 2004: 201).
\end{abstract}

Nuevas y siempre recurridas formas de violencia, violencia de la representación ejercida por los dueños de la palabra, es decir, dueños del conocimiento y de las decisiones políticas. Además de ser los propietarios del capital que será invertido en la solución de los problemas de la pobreza, los discursos profesionales que "interpretan" la realidad, estandarizan las situaciones, las cosifican, las objetivizan, presentándolas como iguales para todas las realidades. "(...) Las categorías determinan el acceso a los recursos (...) la gente tiene que ajustarse a la categorización (...) toda la realidad de una persona queda reducida a un único rasgo o característica (acceso a la tierra, por ejemplo, o a la incapacidad para leer y escribir) (...) la persona se convierte en un caso (...)" (Escobar, 2004: 212). Pareciera que el propósito es obligar a las múltiples realidades sociales a adaptarse a los modelos preestablecidos y sesgados a los intereses de los financiadores del desarrollo.

Así como el desarrollo de las fuerzas productivas en el sistema capitalista hace cada más rico a los ricos y más pobres a los pobres, la economía del mercado y el modelo económico Neoliberal (1980-1990) predominante en los países de América Latina y occidente y 
extendido a otros países del mundo en el contexto de la globalización ha aumentado los niveles de desigualdad social en el mundo, incluido los Estados Unidos de América. Según el Informe Mundial sobre Ciencias Sociales (2016) de CLACSO, el 1\% de la población mundial posee el $50 \%$ de la riqueza de todos los hogares del mundo, para citar solo una cifra, poniendo en evidencia que la "política del goteo" que parte de la falsa creencia de que los países pobres y más vulnerables se benefician del crecimiento económico generado por la fuerza del mercado. La lucha de los países y el nuevo paradigma de desarrollo es ahora en contra la desigualdad social. Este nuevo paradigma desarrollista debe estar presente en planes de desarrollo que no sólo no consultan, sino que desconocen, la realidad diversa, múltiple, rica, cambiante de las comunidades, al constituirse en instrumentos mediáticos de inserción en las formas de vida, se fundamentan en una serie de abstracciones elaboradas por el discurso profesional, que instalado en una supuesta neutralidad, debe garantizar la eficacia del modelo.

Eficacia simbólica, simplemente; toda vez que como señala Escobar: "Las organizaciones dedicadas a la planificación, por ejemplo, siguen un modelo de planeación que se basa en ciertas prácticas que les permiten construir los problemas no sólo de forma que le resulten manejables, sino también eludir la responsabilidad en la implementación del plan" (Escobar, 2004: 214). Y de esta falacia participan, no solo los instrumentalizadores del plan, planificadores y funcionarios públicos; también los gobiernos, las ONG, la academia, los investigadores; instalados en su eficacia profesional. Los únicos excluidos de la formulación del plan y de su implementación discursiva, son los "pobres". Ellos deben consumir el producto, como clientes y consumidores, y reproducir la realidad tal como el poder superior lo establece. Sobre esta eficacia simbólica Escobar ejemplifica con la experiencia de 1971, cuando expertos provenientes del mundo desarrollado, se reunieron en M.IT. para definir la planeación de políticas de alimentación y nutrición para el Tercer Mundo, proponiendo dos estrategias centrales: "la reforma agraria y la llamada revolución verde. Esta última había prometido liberar a la humanidad de la plaga del hambre mediante la aplicación de los últimos hallazgos científicos y tecnológicos en la biología y la agronomía. Su fracaso comenzó a evidenciarse entre comienzos y mediados de la década de los setenta" (Escobar, 2004: 220). Este fracaso se genera básicamente porque en la planeación no hay actores sociales. Hay burócratas, expertos, analistas, consultores, conocedores de los conceptos de la pobreza, pero no conocedores de la realidad de la pobreza. Se deduce la efectividad del plan por sentido común. Haciendo tabla rasa de las condiciones particulares de la vida de los afectados, imponiendo la planetarización propia del capitalismo.

\section{IV}

Sin pretender agotar el análisis del trabajo de Arturo Escobar sobre la invención del Tercer Mundo, miramos con curiosidad, cómo posiblemente estamos girando del paradigma del exterminio de la pobreza gestado desde fines de los años 40 hasta fines de los 80 del siglo anterior, al paradigma de la construcción de la Democracia; y de esta, hacia la disminución de la desigualdad en todas sus dimensiones, pasando de la lucha contra la pobreza y el cumplimiento de los Objetivos del Milenio, hacia la lucha por el fin de la pobreza el cumplimientos de los objetivos de desarrollo sustentables. América Latina necesita Democracia social, comunitaria, participativa. Eso se deduce del informe "La Democracia 
en América Latina. Hacia una Democracia de ciudadanos y ciudadanas" elaborado por Dante Caputo para PNUD en 2004. Y aunque no es el tema del artículo, sí causa curiosidad cómo se va reorientando la brújula del interés internacional hacia otros campos sensibles de la vida latinoamericana.

Dante Caputo señala en su informe que América Latina ha vivido dos décadas de gobiernos democráticos, y a la vez una creciente crisis social, profundas desigualdades, serios niveles de pobreza, crecimiento económico insuficiente, mayor insatisfacción ciudadana. Según los resultados, las raíces de la Democracia en América Latina no son profundas, y por lo tanto el $50 \%$ de los latinoamericanos sacrificarían gobiernos democráticos por mejores condiciones de vida. Y aunque no buscan el regreso al autoritarismo, son demócratas insatisfechos. Podría ser, que con base en la experiencia previa referida al fallido exterminio de la pobreza en el Tercer Mundo, las nuevas estrategias de guerra sean dirigidas hacia el logro de cierto tipo y modelo de Democracia.

Esto refuerza los OBJETIVOS DE DESARROLLO DEL MILENIO reformulados desde 2015 en OBJETIVOS DE DESARROLLO SUSTENTABLES, de la Naciones Unidas: para consolidar la Democracia en América Latina. Quiere decir esto, que la pobreza económica de los países anteriormente descubierto como pobres, es ahora pobreza política, pobreza de Democracia, y que los regimientos de analistas, consultores, investigadores, iniciarán la nueva guerra por la Democracia en América Latina; aun cuando el pueblo latinoamericano no haya analizado y evaluado a profundidad los procesos políticos de Venezuela, Bolivia, Ecuador, Argentina, Nicaragua, Brasil, Paraguay, Uruguay, Costa Rica y la misma Cuba. Hay que adelantarse nuevamente a toda posibilidad de transformación y de apropiación del derecho fundamental de los pueblos de regirse por sus propias determinaciones.

\section{V}

A manera de conclusión se puede plantear que los sistemas culturales proponen sistemas de función simbólica. Se van fundiendo y crean nuevos horizontes. Lo histórico es cultural y es una marca de valor. El valor es inherente, se construye en la cotidianidad, en interrelaciones y prácticas sociales y con la naturaleza. El valor no es trascendente, lo hacemos trascender en el discurso. La narrativa crea al otro. El otro -en este caso el pobre tercermundista- se ha configurado como una víctima: ser careciente de las condiciones que el poder hegemónico capitalista determina.

Las consideraciones referidas de la experiencia de Bután y de Bolivia y Ecuador nos remiten a otras realidades, a otros procesos eminentemente históricos, culturales y étnicos y medio ambientales que integran las dimensiones económicas, políticas, sociales. Estudios realizados por Marcel Mauss, (1971) dan cuenta de esto: el carácter voluntario, libre, gratuito, a la vez obligatorio e interesado de esas prestaciones del presente, del don; la obligatoriedad determinada por el Derecho real, consuetudinario. Las prácticas económicas, la existencia del mercado antes de la aparición de la moneda. José Consuegra Higgins (2003) tiene términos elocuentes al referirse a la economía indígena precolonial de América Latina al catalogarla de ejemplar, autosuficiente basada en la agricultura simple, la caza de animales y la recolección de frutos, donde ya existía la división del trabajo; y el 
comercio se hacía inicialmente mediante el trueque de productos como la sal, el maíz, el algodón, el pescado y el oro, entre otros en mercados locales reducidos y más adelante en mercados más amplios que se operaban en ferias periódicas.

Los que intervienen en el contrato son personas morales: clanes, tribus, familias. No intercambian exclusivamente bienes o riquezas: sobre todo gentilezas, atenciones, festines. El mercado y la circulación es un momento dentro de un proceso más complejo y extenso. Pero no es voluntario y su incumplimiento acarrea una guerra pública o privada. El ejemplo más ilustrativo lo proporciona el Potlach, cuyo propósito es alimentar, consumir, en una mezcla de ritos, prestaciones jurídicas y económicas, fundamentado en tres obligaciones: dar, recibir, devolver.

¿Cuál es la fuerza que obliga a entregar el don? En el potlach no priman los intereses monetarios, mercantilistas, sus elementos fundamentales son el honor, el prestigio, el mana. De la misma manera, Mauss explicita que el taonga de los maorís determina que el bien, el presente, el regalo, el don, posee un poder espiritual. Si la cosa tiene alma, es una obligación entre almas. El pago a los dioses, la limosna, los regalos, se hacen a los niños y a los pobres y agradan a los muertos. Es la vieja moral del don como principio de justicia. La circulación del kula, gran comercio intertribal (Malinowski), constituye otro ejemplo de otras formas de entender la economía y la vida social; así como prácticas no exclusivamente propias de culturas nativas como el diezmo, el haloween, la minga, la tonga, la mano prestada, el convite, etc.

El trueque, el desborde, el gasto, el sacrificio, el fuego como derroche, constituidos como medio de glorificación recíproca (Mauss). Porque no todos los combates se dan en términos de lógica interpretativa, hegemónica, colonizadora, occidentocéntrica, antropocéntrica. Hay otras formas de intercorporalidad intercultural, otras formas de habitar la casa, de construir socialidad, como la música, la gastronomía, la arquitectura, la agricultura, en un marco cósmico, no sólo en un marco de sociabilidad.

La crítica planteada por Escobar en el documento de 2004 analizado inicialmente, toma cuerpo en 2010, en su trabajo Territorios de diferencia: lugar, movimientos, vida, redes, en el cual presenta su propia evaluación sobre las políticas de desarrollo implementadas en el Plan Pacífico, PLAIDECOP y en el Proyecto Biopacífico, en el que contrasta los modelos modernizantes del desarrollo, y presenta sus consideraciones sobre las alternativas a la modernidad, resaltando las consideraciones culturales, no monetaristas de la visión económica tradicional: "(...) La afirmación del ser es una afirmación del derecho a la diferencia, de nuestras culturas, nuestro modo de ser social y nuestra visión de vida. Este principio conduce a que los planes de desarrollo para comunidades y regiones afrocolombianas no sean simplemente planes de inversión, sino canales para potenciar el desarrollo humano.(...)" (Escobar, 2010: 183).

Esto así planteado, suena perfecto, sin embargo, las propuestas y sus resultados dependen del marco conceptual que los conciba, del entramado socio cultural en que se nutra, de los intereses subyacentes o manifiestos que se abran paso a través del discurso. Durante su período presidencial, 1982-1986- el presidente de la época, Belisario Betancur, lo planteó así: 


\begin{abstract}
"Colombia ha ignorado siempre el océano Pacífico, abandonando su litoral a su suerte. El gobierno desea dedicar los cuatro años de su período presidencial en establecer los cimientos para una gran política para el Pacífico y la elaboración de su primer plan de desarrollo" (DNP-CVCUNICEF 1983: 13). Fue entonces que el Pacífico apareció completamente como una entidad desarrollable, con consecuencias dramáticas. (...) (Escobar, 2010: 187)
\end{abstract}

Habría que decir contrariando al presidente Betancur, que lo ignorado siempre no fue el océano Pacífico, sino las comunidades asentadas en las costas y riberas de los ríos que desembocan en el Pacífico, seres de carne y hueso, con sentimientos y recursividades para enfrentar el abandono estatal; excluidas sistemáticamente de los sistemas de integración económicos y sociales; fue la refrendación de la invisibilización que se construyó en la Colonia.

Sin embargo, según Escobar proliferaron los foros nacionales e internacionales para darle "materialidad" a la nueva orientación, el "descubrimiento" del Pacífico, y los consecuentes debates entre quienes defienden la modernidad como camino, versus quienes propugnan por la concepción de modernidades alternativas, hasta las propuestas de "(...) alternativas a la modernidad, como un proyecto más radical y visionario de redefinir y reconstruir mundos locales y regionales desde la perspectiva de las prácticas de la diferencia cultural, económica y ecológica, siguiendo una lógica de la red y en contextos de poder." (Escobar, 2010: 188)

Escobar se pregunta ¿qué está pasando con el desarrollo?: nada nuevo. Es la reedición del modelo de representación impuesto desde la Colonia. El desconocimiento persistente de la capacidad de creación de los "otros", negros e indígenas. La desviación del concepto de planeación del desarrollo hacia la implementación de planes concebidos en otras latitudes, en otros contextos:
"Para las comunidades negras e indígenas el término "biodiversidad" viene desde el exterior; no es usado por las personas. Las comunidades negras han usado el monte (literalmente, "zona rural") en más o menos la misma manera que lo que implica la palabra de biodiversidad, ya que es en el monte donde las formas diversas de la vida son encontradas. La relación entre naturaleza (territorio), la cultura y la cotidianidad es igual a la biodiversidad. Los saberes de las comunidades negras e indígenas han permitido una relación armónica con el ambiente, favoreciendo la diversidad biológica" (Escobar, 2010: 212).

Es la imposición de esquemas de análisis, de discursos preelaborados; es la negación de la capacidad de las comunidades para entenderse, asumirse, empoderarse, construirse permanentemente; estar en el mundo como ellos han querido o han podido establecerse sin el concurso de los "otros". Es la instauración del reino de la indignidad, de la inmadurez intelectual y de la incapacidad política. Es el mesianismo reformulado; la evangelización remozada; el colonialismo académico revestido de buenas intenciones y neutralidades morales.

Es el desconocimiento de la dimensión social integral de las comunidades; es la reducción de la planeación a inventariar las necesidades básicas insatisfechas de orden material: vivienda, alimento y vestido: 
Pero en ningún momento se considera como punto de partida para la planificación, la POTENCIALIDAD SOCIAL de la comunidad, lo cual implica: los recursos naturales, el recurso humano, pero también los saberes, oficios, profesiones, el imaginario de la comunidad, la tradición, los intereses de clase, la cultura, la vivencia política, las expectativas, los sueños, etc., sin los cuales los planificadores no resolverían el interrogante: ¿CÓMO HA LOGRADO SOBREVIVIR LA POBLACIÓN? (Eljach, 1992: 59).

Significa poner el énfasis en la torsión simbólica gramsciana, desarmar los discursos autorizados para recuperar el lenguaje natural de los pueblos, abrir otros diálogos, abrir otras lenguas, otras escrituras; se requiere romper el espacio-tiempo otro del ciclo discursivo dominante; por cuanto es en la materialidad cultural, en la corporalidad cultural, donde se relativizan y rompen los universalismos, se destruye el homologismomonologismo universal de la modernidad.

En la práctica, en Colombia tuvieron que transcurrir más de 140 años desde la abolición legal de la esclavización para que los afrocolombianos fueran reconocidos por el Estado como un grupo étnico y como tal sujetos de derechos colectivos e individuales; esto se produce en la Constitución Política de 1991 que reconoce a Colombia como Estado pluriétnico y pluricultural y con ello la obligación estatal de proteger la diversidad étnica y cultural del país. Y más adelante, aunque mediante la figura del Artículo Transitorio 55, le impone la obligación al Congreso de expedir una Ley que haga referencia en forma exclusiva a los afrocolombianos y raizales de San Andrés y Providencia.

Como producto de lo consignado en el Artículo Transitorio 55, y de los mandatos constitucionales de que Colombia es un Estado social de Derecho, pluralista, fundado en el respeto de la dignidad humana, en el que el Estado mismo reconoce y protege la diversidad étnica y cultural de la nación colombiana, el Congreso expidió la Ley 70 del 27de Agosto de 1993.

El propósito fundamental de la Ley 70 es acabar con la "invisibilidad" institucional y legal de la etnia afro en Colombia; anteriormente su reconocimiento fue negativo, discriminatorio. Su propósito es reconocer a las comunidades "negras" que han venido ocupando tierras baldías en las zonas rurales ribereñas de los ríos de la cuenca del Pacífico y de otras zonas del país, de acuerdo a sus prácticas tradicionales de producción, el derecho a la propiedad colectiva, de acuerdo a los requisitos establecidos por la misma ley. Así como establecer mecanismos para la identidad cultural y los derechos de las comunidades "negras" como grupo étnico, y el fomento de su desarrollo social y económico, para garantizarles condiciones reales de igualdad de oportunidades frente al resto de la población colombiana.

Sin embargo, los territorios colectivos de las comunidades "negras", no fueron elevados a la condición de Entidades Territoriales, tal como sucedió con los pueblos indígenas de Colombia. Por lo tanto, lo establecido en la Constitución Política de 1991, no tiene hasta el momento, ninguna viabilidad operativa para el reconocimiento jurídico, social y político, solo tiene un efecto simbólico. 
Desvelar la eficacia simbólica del discurso oficial, sea de la planeación o de cualquier otra dimensión, plantea el reto de entender el desarrollo como un producto social, histórico y cultural, matizado de diversidad de texturas convergentes en una polisemia de sentidos, desentrañables para los conocedores de la palabra y del sentido de la misma, pero inextricables para la inmensa mayoría de las personas, para quienes la pobreza, el hambre, la vida, tienen una significación cultural; aspecto precisamente negado por los interpretadores de su situación.

Los sistemas históricos, económicos, políticos, y culturales proponen y proyectan sistemas de función simbólica. Se van fundiendo y crean nuevos horizontes y paradigmas, sin tener en cuenta al otro: el malnutrido, al pobre al tercermundista como víctima careciente de las condiciones que el poder hegemónico capitalista determina. La lucha contra la pobreza, el cumplimiento de los Objetivos del Milenio y los Objetivos de Desarrollo Sustentable de las Naciones Unidas y muy recientemente la lucha contra las distintas dimensiones de la desigualdad social, del Informe 2016 de CLACSO, demostrarán que la retórica desarrollista en el discurso, se impone en este caso.

Consideraciones resaltadas de la experiencia de Bután y de Bolivia y Ecuador nos remiten a otras realidades, a otros procesos eminentemente históricos, culturales, étnicas y medio ambientales que integran las dimensiones económicas, políticas, sociales. Lo histórico es cultural y es una marca de valor. El valor es inherente, se construye en la cotidianidad, en interrelaciones y prácticas sociales y con la naturaleza. El valor no es trascendente, lo hacemos trascender en el discurso. La narrativa crea al otro.

Estudios realizados por Marcel Mauss, (1971) dan cuenta de esto: el carácter voluntario, libre, gratuito, a la vez obligatorio e interesado de esas prestaciones del presente, del don; la obligatoriedad determinada por el Derecho real, consuetudinario. Las prácticas económicas, la existencia del mercado antes de la aparición de la moneda.

El concepto occidental de desarrollo aun persiste y se sigue interpretando como el resultado de políticas económicas aplicadas en los países, desconociendo que el desarrollo abarca no solo dimensiones económicas, sino dimensiones subjetivas de inclusión, de prácticas del "Buen Vivir", de participación de todos actores sociales y el respeto por el medio ambiente. En este sentido los nuevos indicadores de medida del desarrollo están dando un viraje al tener en cuenta variables no solo de carácter económico sino de impacto subjetivo y ambiental.

El discurso de la narrativa no solo debe incluir al otro sino también a la naturaleza misma y a los hijos de la Pachamama recogiendo su acervo cultural, histórico para introducir modificaciones en la hegemonía del pensamiento eurocéntrico y crear nuevas prácticas de pensamiento para poder interpretar mejor las realidades socioeconómicas de América Latina, Nuestra América, y del Mundo. 


\section{REFERENCIAS BIBLIOGRÁFICAS}

Anderson, P. (1998) Las antinomias de Antonio Gramsci. Estado y revolución en Occidente. México: Fontamara (1977).

Augé, M. (1996) El sentido de los otros. Paidós, Barcelona.

Caputo, D. (2004) La Democracia en América Latina. Hacia una democracia de ciudadanas y ciudadanos. PNUD.

CLACSO. Informe Mundial sobre Ciencias Sociales (2016). Afrontar el Reto de las Desigualdades y trazar vías Hacia un mundo justo.

Congreso de la república (1993) Ley 70 de agosto 27 de 1993. Por la cual se reglamenta el Artículo Transitorio 55 de la Constitución Política de Colombia.

Consuegra, J. (2003) El pensamiento económico colombiano. Editorial Corporación Educativa Mayor del Desarrollo Simón Bolívar

Eljach, M. (1992) Dimensión social y política de la planificación (Ensayo). Revista Administración y Desarrollo N 30 junio de 1992, ESAP. Bogotá, pp. 56-66.

(2006) La construcción jurídica del negro en la Colonia. De Benkos Biohó a la invisibilidad. AXIS MUNDI, Popayán.

Escobar, A. (2004) "La problematización de la pobreza: la fábula de los tres mundos y el desarrollo", "El manejo del cambio social: la constitución de la planeación del desarrollo", "La dispersión del poder: fábulas del hambre y alimento". En: La invención del Tercer Mundo. Construcción y deconstrucción del desarrollo. Norma, pp. 51-111, 170-294.

(2010) Territorios de diferencia: lugar, movimientos, vida, redes. Popayán: Envión.

Foucault, M. (1999) El orden del discurso. Tusquets, Barcelona.

Gudynas E. (2011) Buen Vivir: Germinado alternativas al desarrollo. América Latina en Movimiento

Guimón, P. (2009) El reino que quiso medir la felicidad. www.elpaís.com noviembre 29 de 2009. elpais.com/diario/2009/11/29/eps/1259479614_850215.html

INFORME BRUNDTLAND 1987 presentado por la Misión de Medio Ambiente y desarrollo de la ONU conocido «Nuestro futuro común» (más comúnmente denominado por el nombre del Presidente de la Comisión, Gro Harlem Brundtland, Primer Ministro de Noruega.

Malinowski, B. (2001) Los argonautas del Pacífico occidental. Comercio y aventura entre los indígenas de la Nueva Guinea melanésica. Barcelona: Península (1922).

Mancero X. (2001) La medición del desarrollo humano: elementos de debate. Documento CEPAL

Mauss, M. (1971) Ensayo sobre los dones: Razón y forma del cambio en las sociedades primitivas. (1923). Publicado en Sociología y Antropología, Madrid, Editorial Tecnos.

Naciones Unidas. (1987) “Nuestro Futuro Común” En: Informe Brundtland.

Naciones Unidas.) (2015 “Los Objetivos de desarrollo sustentables”.

PNUD. Informe de Desarrollo Humano (1990). Tercer Mundo Editores Bogotá Colombia. 
República de Colombia (1991) Constitución Política de Colombia, julio 4 de 1991.

Santos, B de S. (2011). Epistemologías del sur. Utopía y Praxis Latinoamericana / Año 16. Nº 54 (JulioSeptiembre, 2011) Pp. 18

Sen A. (2000) Desarrollo y Libertad. Editorial Tapa Blanda

Thompson, E. P. (1995) Costumbres en común. Barcelona: Crítica (1991).

\section{Para citaciones:}

Eljach, M., y Quiroz, E. (2018). La eficacia simbólica del desarrollo: Estrategia de construcción del sujeto histórico. Panorama Económico, 26(4), 497-514.

\section{AUTORES}

Matilde Eljach

Doctora y Magister en Antropología de la Universidad del Cauca (Colombia). Especialista en Administración de la Planeación Urbana y Regional (ESAP-Universidad del Cauca). Cuenta con experiencia docente en la Universidad del Cauca (Colombia) y actualmente, Docente investigadora de la Universidad Simón Bolívar (Colombia), e integrante del Centro de Investigación e Innovación Social José Consuegra Higgins, Grupo de Investigación Estudios Interdisciplinarios sobre el Caribe

\section{Ezequiel Quiroz}

Economista, Grupo de Investigación Estudios Interdisciplinarios del Caribe de la Universidad Simón Bolívar de Barranquilla. 